\title{
Ground-State Phase Diagram of a Model FCC Magnet with Modified RKKY Interaction
}

\author{
K. Szalowski And T. BALCERZAK \\ Department of Solid State Physics, University of Łódź \\ Pomorska 149/153, 90-236 Eódź, Poland
}

\begin{abstract}
The ground-state phase diagram is studied for a diluted model magnet with FCC structure and modified Ruderman-Kittel-Kasuya-Yosida interaction. The modification consists in taking into account the exponential damping for the long-range interaction as well as the nearest-neighbors antiferromagnetic superexchange couplings. The existence of paramagnetic, ordered ferromagnetic and three distinct antiferromagnetic phases is shown. The stability areas of the phases upon the free-carriers concentration are investigated.
\end{abstract}

PACS numbers: 75.50.Pp, 75.10.-b, 75.30.Hx, 75.30.Kz

\section{Introduction}

A wide interest in application of the dilute magnetic semiconductors (DMS), such as $\mathrm{Ga}_{1-x} \mathrm{Mn}_{x} \mathrm{As}$, is stimulating for investigations of the models based on the Ruderman-Kittel-Kasuya-Yosida (RKKY) interaction [1, 2]. It follows from the experimental works [3] that the studies of the magnetic phase diagrams are of primary importance. However, in order to describe the magnetic properties of DMS, the free-carriers localization as well as the nearest-neighbors (NN) antiferromagnetic interaction should be taken into account. Such a model has been exploited in [2], where the existence of ferromagnetic phase has been studied by Monte Carlo (MC) method in some range of small concentrations of the free carriers. However, as it has been stated in [2], the MC method was unable to distinguish between the non-ferromagnetic phases existing for higher concentrations. This fact encouraged us to undertake the studies of the complete ground-state phase diagram for the diluted FCC magnet with modified RKKY interaction. For this purpose, the numerical method of near-exact calculation of the energy in the ground state is adopted [4]. It is shown that the modification of the RKKY interaction results in a rich structure of the phase diagram in a wide range of the free-carriers concentration. 


\section{Theoretical model}

We consider a diluted magnet with FCC structure described by the Ising-like Hamiltonian, where only the $z$-components of the spins $S$ are taken into account

$$
\mathcal{H}=-\sum_{\langle i, j\rangle} J^{\mathrm{RKKY}}\left(r_{i j}\right) \xi_{i} \xi_{j} S_{i}^{z} S_{j}^{z}-\sum_{\langle i, j\rangle^{\prime}} J^{\mathrm{AF}} \xi_{i} \xi_{j} S_{i}^{z} S_{j}^{z}
$$

The first term in Eq. (1) stands for the RKKY long-range interaction, whereas the second term describes the antiferromagnetic superexchange NN couplings. The occupation operators $\xi_{j}=0,1$ are responsible for the site-dilution, and their configurational averages are interpreted as the magnetic ions concentrations: $n_{i}=\left\langle\xi_{j}\right\rangle_{r}$. The RKKY exchange integral is given by the formula [5]:

$$
J^{\mathrm{RKKY}}(r)=C\left(k_{\mathrm{F}} a\right)^{4}\left[\sin \left(2 k_{\mathrm{F}} r\right)-2 k_{\mathrm{F}} r \cos \left(2 k_{\mathrm{F}} r\right)\right]\left(2 k_{\mathrm{F}} r\right)^{-4} \mathrm{e}^{-r / \lambda} .
$$

The integral contains the exponential damping factor, parameterized by the characteristic length scale $\lambda$, which arises from the free-carriers localization [5]. For the FCC structure with lattice constant $a$ the Fermi wave vector is given by $k_{\mathrm{F}}=\left(12 \pi^{2} n_{\mathrm{c}}\right)^{1 / 3} / a$, where $n_{\mathrm{c}}$ is the free-carriers concentration (a number of the carriers per one lattice site). The energy constant $C$ has the form $C=$ $2 A^{2} m^{*} /\left(\pi h^{2} a^{4}\right)$ and depends on the effective mass $m^{*}$ and the contact potential $A$ for the carriers.

In the ground state (for $T=0$ ) we consider the existence of disordered paramagnetic $(\mathrm{P})$ phase as well as the ferromagnetic $(\mathrm{F})$ phase and three antiferromagnetic $(\mathrm{AF})$ : first kind ( $\mathrm{AF} 1)$, first kind improved $(\mathrm{AF} 1 \mathrm{I})$ and second kind (AF2) phases [6]. For each phase the ground-state energy per one lattice site $U / N=\langle\langle\mathcal{H}\rangle\rangle_{r} / N$ can be written in the form

$$
U / N=-\frac{1}{2} n_{i}^{2} S^{2}\left[\sum_{k}\left(z_{k}^{\uparrow \uparrow}-z_{k}^{\uparrow \downarrow}\right) J^{\mathrm{RKKY}}\left(r_{k}\right)+\left(z_{1}^{\uparrow \uparrow}-z_{1}^{\uparrow \downarrow}\right) J^{\mathrm{AF}}\right],
$$

where by $z_{k}^{\uparrow \uparrow}\left(z_{k}^{\uparrow \downarrow}\right)$ we denote the number of spins lying on the $k$-th coordination zone and oriented parallel (antiparallel) to the central spin. These numbers are characteristic of the given phase and can be found numerically. The expression for $U / N$ is scaled by the arbitrary dilution $n_{i}$ and spin $S$, and has been used for the phase diagram calculation.

\section{Numerical results and discussion}

The numerical calculations have been carried out for the long-range interaction extending up to $100 a$ which corresponds to 18335 coordination zones in FCC structure. By comparison of the ground-state energies (3) the stability regions for each phase have been found.

In Fig. 1 we present the stability areas in the $\left(n_{\mathrm{c}}, \lambda / a\right)$-coordinates for (a) $J^{\mathrm{AF}}=0$ and (b) $J^{\mathrm{AF}} / C=-0.5$. A variety of different phases depending on the free-carriers concentration is shown. It is visible in Fig. 1a that the influence of the damping for pure RKKY interaction manifests itself mainly for $\lambda / a \leq 1$. 

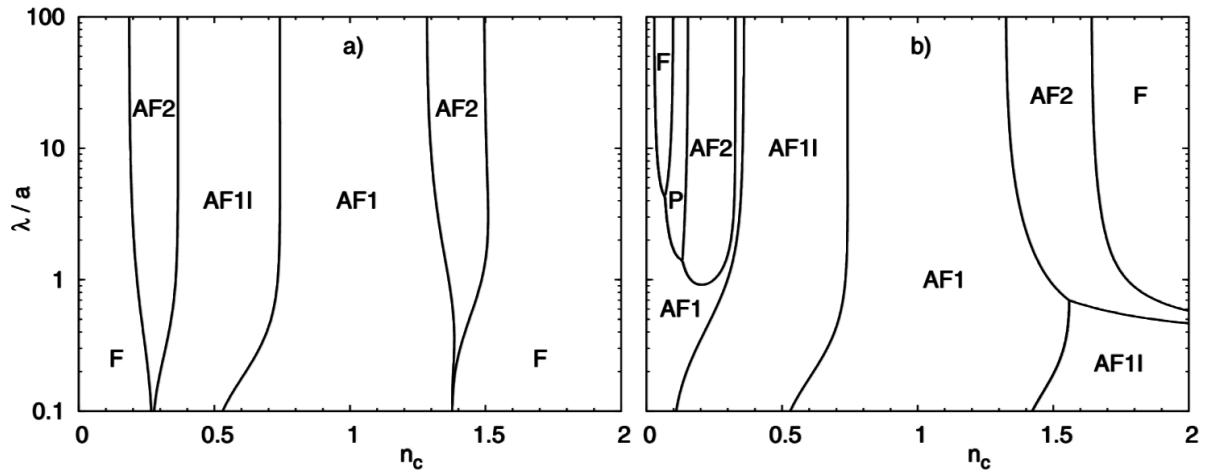

Fig. 1. The ground-state phase diagram in $\left(n_{\mathrm{c}}, \lambda / a\right)$-coordinates for (a) $J^{\mathrm{AF}}=0$ and (b) $J^{\mathrm{AF}} / C=-0.5$.

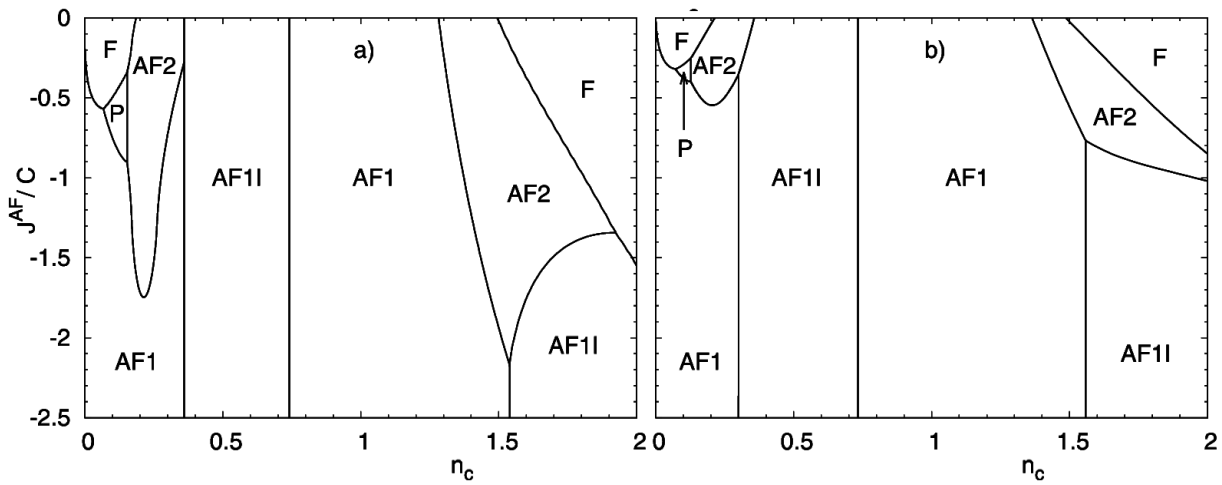

Fig. 2. The ground-state phase diagram in $\left(n_{\mathrm{c}}, J^{\mathrm{AF}} / C\right)$-coordinates for (a) $\lambda / a \rightarrow \infty$ and (b) $\lambda / a=1$.

A presence of antiferromagnetic interaction in Fig. 1b essentially changes the diagram. For small concentrations $n_{\mathrm{c}}$ the F-phase exists in a narrow range only, and the paramagnetic phase emerges. The influence of damping in Fig. $1 \mathrm{~b}$ is already noticeable for $\lambda / a \leq 10$. The damping of RKKY interaction in Fig. $1 \mathrm{~b}$ reduces the F-phase. Figure 1a, by contrast, has F-areas becoming larger with the corresponding decrease in $\lambda$.

In Fig. 2 the phase diagram in $\left(n_{\mathrm{c}}, J^{\mathrm{AF}} / C\right)$-coordinates is presented (a) for $\lambda / a \rightarrow \infty$ (i.e., without the RKKY damping) and (b) for a relatively strong damping with $\lambda / a=1$. In particular, in Fig. 2a the phase boundaries for the line $J^{\mathrm{AF}}=0$ are in agreement with the results of the paper [4]. It also follows from Fig. 2a that the existence of $\mathrm{P}$-phase is possible, but in a restricted range of $J^{\mathrm{AF}}$-parameters. In the case of strong damping (Fig. 2b) both F, AF2, and P-regions are more confined than in Fig. 2a, and all changes are most evident for small values of $J^{\mathrm{AF}}$. In both figures (1 and 2$)$ the existence of the second region of F-phase (for large $n_{\mathrm{c}}$ values) is worth noticing. 
In conclusion, a rich ground-state phase diagram has been found for the presented model. The observations concerning the F-phase behavior for small concentrations are in agreement with MC simulations [2]. The validity of the presented approach can be discussed in context of the percolation threshold for the damped RKKY interaction, for $\lambda \rightarrow 0$. The magnetization of the charge carriers is also well worth considering in further developments.

\section{Acknowledgments}

This work was supported by the European Social Fund and Budget of State implemented under the Integrated Regional Operational Program, action 2.6, project GRRI-D.

\section{References}

[1] T. Dietl, A. Haury, Y. Merle d'Aubigné, Phys. Rev. B 55, R3347 (1997).

[2] D.J. Priour, Jr., S. Das Sarma, Phys. Rev. Lett. 97, 127201 (2006).

[3] O.M. Fedorych, Z. Wilamowski, M. Potemski, M. Byszewski, J. Sadowski, Semicond. Sci. Technol. 19, S492 (2004).

[4] T. Balcerzak, J.W. Tucker, A. Bobák, M. Jaščur, Czech J. Phys. 54, D643 (2004).

[5] D.C. Mattis, The Theory of Magnetism I, Springer-Verlag, Berlin 1981.

[6] A.H. Morrish, The Physical Principles of Magnetism, Wiley, New York 1965. 